\title{
DIFFERENTIAL CALCULUS ON A THREE-PARAMETER OSCILLATOR ALGEBRA
}

\author{
Michèle IRAC-ASTAUD * \\ Laboratoire de Physique Théorique et Mathématique \\ Université Paris VII \\ 2 place Jussieu F-75251 Paris Cedex 05, FRANCE
}

to be published in Reviews in Mathematical Physics

\begin{abstract}
Two differential calculi are developped on an algebra generalizing the usual q-oscillator algebra and involving three generators and three parameters. They are shown to be invariant under the same quantum group that is extended to a ten-generator Hopf algebra. We discuss the special case where it reduces to a deformation of the invariance group of the Weyl-Heisenberg algebra for which we prove the existence of a constraint between the values of the parameters.
\end{abstract}

\section{Introduction}

Generalizing the differential geometry on Lie groups and manifolds, the differential calculus on quantum groups and quantum spaces was developed in many interesting papers, (see for example [1], [2], [3], [4] ). An exterior differential, one-forms and partial derivatives are defined on a quadratic algebra, that is, a free associative algebra generated by variables satisfying quadratic commutation relations. In the present paper, as in [10] and [11], we consider

*e-mail : mici@ccr.jussieu.fr 
the physically important case where the quadratic algebra is a deformation of the Weyl-Heisenberg algebra, in order to obtain a differential calculus on the algebra of the observables of a quantum system.

The Weyl-Heisenberg algebra can be seen as a free associative algebra generated by three variables $x^{i}$ satisfying the quadratic relations :

$$
R:\left\{\begin{aligned}
x^{1} x^{2}-x^{2} x^{1}-s\left(x^{3}\right)^{2} & =0 \\
x^{1} x^{3}-x^{3} x^{1} & =0 \\
x^{2} x^{3}-x^{3} x^{2} & =0
\end{aligned}\right.
$$

where $\hat{a}=x^{1}, \quad \hat{a}^{\dagger}=x^{2}$ and $x^{3}$ is the identity. This algebra is denoted by $C<x>/ R$.

This space is invariant under the seven-dimensional Lie subgroup of $G L(3)$, $G$, constituted by the matrices $T$ such as $t_{1}^{3}=t_{2}^{3}=0$ and $t_{1}^{1} t_{2}^{2}-t_{2}^{1} t_{1}^{2}=\left(t_{3}^{3}\right)^{2}$.

The quadratic deformation of the Weyl-Heisenberg algebra considered in this paper, as in [10] and [11], is an algebra, generalizing $C<x>/ R$, obtained by replacing the relations (1) by

$$
R_{x x}:\left\{\begin{aligned}
x^{1} x^{2}-q x^{2} x^{1}-s\left(x^{3}\right)^{2} & =0 \\
x^{1} x^{3}-u x^{3} x^{1} & =0 \\
x^{2} x^{3}-u^{-1} x^{3} x^{2} & =0
\end{aligned}\right.
$$

When $q=u^{-2}$, putting $x^{1}=\hat{a}, \quad x^{2}=\hat{a}^{\dagger}$ and $x^{3}=q^{-\frac{N}{2}}$, the algebra $C\left\langle x>/ R_{x x}\right.$ is identified to the q-oscillator algebra [8] [9]. When $s=0$, $C<x>/ R_{x x}$ is the three-dimensional quantum plane [5].

In [10] and [11], we establish that a differential calculus on $C<x>/ R_{x x}$ invariant under a seven-dimensional quantum group, deformation of $G$, only exists if $q=u^{2}$ and then is unique. As the constraint, $q=u^{2}$, eliminates the important case when the quantum space is the q-oscillator algebra, the aim of this paper is to obtain a differential calculus on $C<x>/ R_{x x}$ not restricted, a priori, by a requirement of invariance and valid for arbitrary values of the deformation parameters $q, u$ and $s$.

In section 2, we determine two different sets of consistent quadratic relations between variables, differentials and derivatives. In section 3 , we prove that all these relations are invariant under the same quantum group that is extended to a ten-generator Hopf-algebra. When we assume that the variables $x^{1}$ and $x^{2}$ are mutually adjoint and that $x^{3}$ is self-adjoint, the quantum group is endowed with a structure of Hopf-star-algebra. Finally we discuss 
the particular case where the quantum group is a deformation of $G$ and we recover the constraint on the parameters [10], [11]. In this particular case, the ten-generator Hopf-star-algebra contains a eight-generator subalgebra, both algebras leave invariant the commutation relations defining the differential calculus.

\section{Differential Calculus}

Following the usual method [3] [4], we add to the free algebra $C\langle x\rangle$, three generators $\xi^{i}, i=1,2,3$, identified to the one-forms. We define the exterior differential operator $d$ in $C<x, \xi>$ such as :

$d\left(x^{i}\right)=\xi^{i}$,

$d$ is linear,

$d^{2}=0$

and $d$ satisfies the graded Leibniz rule :

$$
d(f g)=(d f) g+(-1)^{k} f(d g)
$$

where $f, g \in C<x, \xi\rangle$ and $f$ is of degree $k$. Then, the partial derivatives $\partial_{i}$ are defined by :

$$
d \equiv \xi^{i} \partial_{i}
$$

The commutation relations $R_{x \xi}$ between the variables and the differentials and those between the partial derivatives $\partial_{k}$ and the forms $\xi^{l}$ are assumed to be quadratic [3] [胃:

$$
R_{x \xi} \quad: \quad x^{k} \xi^{l}=C_{m n}^{k l} \xi^{m} x^{n}
$$

and

$$
R_{\partial \xi} \quad: \quad \partial_{k} \xi^{l}=K_{k n}^{l m} \xi^{n} \partial_{m}
$$

By applying operator $d$ to (四) on the left, we get :

$$
R_{\xi \xi}: \xi^{k} \xi^{l}=-C_{m n}^{k l} \xi^{m} \xi^{n},
$$

The above definition implies : $\partial_{l}\left(x^{k}\right)=\delta_{l}^{k}$. Applying the Leibniz rule on $x^{k} f, f \in C<x>$, and taking into account relations (幽, we obtain the commutation relations between $\partial_{i}$ and $x^{k}$ [3]:

$$
R_{x \partial} \quad: \quad \partial_{l} x^{k}=\delta_{l}^{k}+C_{l n}^{k m} x^{n} \partial_{m}
$$


The combinations of the relations $R_{\partial \xi}, R_{x \partial}$ and $R_{x \xi}$ lead to

$$
K=C^{-1}
$$

and to a sufficient condition of consistency, the Yang-Baxter equation :

$$
(C \otimes 1)(1 \otimes C)(C \otimes 1)=(1 \otimes C)(C \otimes 1)(1 \otimes C)
$$

where 1 is the identity of $G L(3)$.

From this point, we simplify the matrix $C$ assuming that $C_{l m}^{i j}$ is zero if $(i, j) \neq(l, m)$ or $(m, l)$ except $C_{33}^{12}$ and $C_{33}^{21}$. We multiply the relations $R_{x x}$ on the left by $\partial_{i}$. Using $R_{x \partial}$, we commute $\partial_{i}$ to the right, and obtain several relations between the elements $C_{k l}^{i j}$. In particular,

$$
\begin{array}{llrl}
C_{12}^{12} & =q C_{12}^{21}-1, & & C_{21}^{12}=q C_{21}^{21}+q \\
C_{13}^{13} & =u C_{13}^{31}-1, & & C_{31}^{13}=u C_{31}^{31}+u \\
C_{23}^{32}=u C_{23}^{23}+u, & C_{32}^{32} & =u C_{32}^{23}-1 \\
C_{33}^{12} & =q C_{33}^{21}+s C_{33}^{33}+s & &
\end{array}
$$

and

$$
C_{12}^{12} C_{21}^{21}=0, \quad C_{13}^{13} C_{31}^{31}=0, \quad C_{23}^{23} C_{32}^{32}=0
$$

We substitute in the equation (9) a matrix $C$ satisfying (10). This gives $27 \times 27$ new relations between the coefficients $C_{k l}^{i j}$. Solving these relations together with (11), we find only two different solutions : $C$ is equal to $\Omega$ or to its inverse, with $\Omega$ defined by :

$\Omega=\left(\begin{array}{ccccccccc}q / u^{2} & 0 & 0 & 0 & 0 & 0 & 0 & 0 & 0 \\ 0 & 0 & 0 & q^{2} / u^{2} & 0 & 0 & 0 & 0 & q s / u^{2} \\ 0 & 0 & 0 & 0 & 0 & 0 & q / u & 0 & 0 \\ 0 & q^{-1} & 0 & q / u^{2}-1 & 0 & 0 & 0 & 0 & -s / q \\ 0 & 0 & 0 & 0 & q / u^{2} & 0 & 0 & 0 & 0 \\ 0 & 0 & 0 & 0 & 0 & q / u^{2}-1 & 0 & 1 / u & 0 \\ 0 & 0 & 1 / u & 0 & 0 & 0 & q / u^{2}-1 & 0 & 0 \\ 0 & 0 & 0 & 0 & 0 & q / u & 0 & 0 & 0 \\ 0 & 0 & 0 & 0 & 0 & 0 & 0 & 0 & q / u^{2}\end{array}\right)$

The matrices $\Omega$ and $\Omega^{-1}$ have the same eigenspaces that correspond to the variable quantum space defined by $R_{x x}$ and to the one-form quantum space 
defined by :

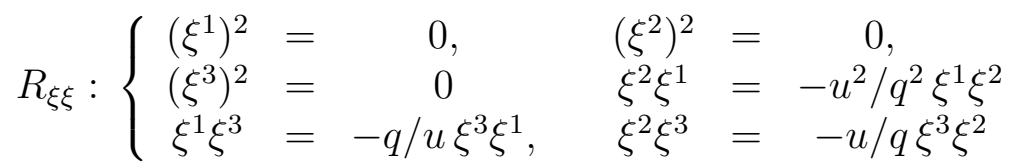

When $C$ is equal to $\Omega$ and to its inverse, the eigenspaces of the transpose matrix $\left(C^{-1}\right)^{t}$ are the same. The six-dimensional eigenspace is identified to the derivative quantum space and is defined by :

$$
R_{\partial \partial}:\left\{\begin{array}{l}
\partial_{1} \partial_{2}=u^{2} / q^{2} \partial_{2} \partial_{1} \\
\partial_{1} \partial_{3}=u / q \partial_{3} \partial_{1} \\
\partial_{2} \partial_{3}=q / u \partial_{3} \partial_{2}
\end{array}\right.
$$

The three-dimensional eigenspace corresponds to the covariant differential forms. We denote $R$ the set of relations (2), (13) and (14). Corresponding to $C=\Omega$ or $C=\Omega^{-1}$, we obtain two sets of relations $R_{x \xi}, R_{\partial \xi}$ and $R_{\partial x}$ and then two different differential calculi :

- The set of relations $R^{\Omega}$ associated to $\Omega$ is :

The commutation relations $R_{x \xi}^{\Omega}$ between the variables and the differentials :

$$
\begin{aligned}
& x^{i} \xi^{i}=q / u^{2} \xi^{i} x^{i}, \quad i=1,2,3, \quad x^{1} \xi^{3}=q / u \xi^{3} x^{1}, \\
& x^{1} \xi^{2}=q^{2} / u^{2} \xi^{2} x^{1}+q s / u^{2} \xi^{3} x^{3}, \quad x^{3} \xi^{2}=q / u \xi^{2} x^{3} \text {, } \\
& x^{2} \xi^{3}=\left(q / u^{2}-1\right) \xi^{2} x^{3}+1 / u \xi^{3} x^{2}, \quad x^{3} \xi^{1}=\left(q / u^{2}-1\right) \xi^{3} x^{1}+1 / u \xi^{1} x^{3} \\
& x^{2} \xi^{1}=1 / q \xi^{1} x^{2}+\left(q / u^{2}-1\right) \xi^{2} x^{1}-s / q \quad \xi^{3} x^{3} \text {, }
\end{aligned}
$$

The commutation relations $R_{\partial \xi}^{\Omega}$ between the derivatives and the differentials :

$$
\begin{aligned}
& \partial_{3} \xi^{3}=\left(u^{2} / q-1\right) \xi^{2} \partial_{2}+u^{2} / q \xi^{3} \partial_{2} \quad \partial_{1} \xi^{2} \quad=u^{2} / q^{2} \xi^{2} \partial_{1}, \\
& \partial_{1} \xi^{3}=u / q \xi^{3} \partial_{1}, \quad \partial_{2} \xi^{1} \quad=q \xi^{1} \partial_{2}, \\
& \partial_{3} \xi^{2}=u / q \xi^{2} \partial_{3}-s u^{2} / q^{2} \xi^{3} \partial_{1}, \quad \partial_{2} \xi^{3} \quad=u \xi^{3} \partial_{2}, \\
& \partial_{3} \xi^{1}=u \xi^{1} \partial_{3}+s \xi^{3} \partial_{2}, \quad \partial_{2} \xi^{2} \quad=u^{2} / q \xi^{2} \partial_{2} \text {, } \\
& \partial_{1} \xi^{1}=u^{2} / q \xi^{1} \partial_{1}+\left(u^{2} / q-1\right) \xi^{3} \partial_{3}+\left(u^{2} / q-1\right) \quad \xi^{2} \partial_{2},
\end{aligned}
$$

The commutation relations $R_{x \partial}^{\Omega}$ between the derivatives and the variables :

$$
\begin{aligned}
& \partial_{1} x^{1}=1+q / u^{2} x^{1} \partial_{1}, \quad \partial_{2} x^{3}=q / u x^{3} \partial_{2}, \\
& \partial_{3} x^{3}=1+q / u^{2} x^{3} \partial_{3}+\left(q / u^{2}-1\right) x^{1} \partial_{1}, \quad \partial_{1} x^{2}=1 / q x^{2} \partial_{1}, \\
& \partial_{3} x^{1}=q / u x^{1} \partial_{3}+q s / u^{2} x^{3} \partial_{2}, \quad \partial_{2} x^{1} \quad=q^{2} / u^{2} x^{1} \partial_{2}, \\
& \partial_{3} x^{2}=1 / u x^{2} \partial_{3}-s / q x^{3} \partial_{1}, \quad \partial_{1} x^{3}=1 / u x^{3} \partial_{1} \text {, } \\
& \partial_{2} x^{2}=1+q / u^{2} x^{2} \partial_{2}+\left(q / u^{2}-1\right) \quad x^{1} \partial_{1}+\left(q / u^{2}-1\right) x^{3} \partial_{3} \text {. }
\end{aligned}
$$


- The set of relations associated to $\Omega^{-1}$ :

The commutation relations $R_{x \xi}^{\Omega^{-1}}$ between the variables and the differentials :

$$
\begin{aligned}
& x^{i} \xi^{i}=u^{2} / q \xi^{i} x^{i}, \quad i=1,2,3, \quad x^{1} \xi^{3} \quad=\left(u^{2} / q-1\right) \xi^{1} x^{3}+u \xi^{3} x^{1}, \\
& x^{3} \xi^{1}=u / q \xi^{1} x^{3}, \quad x^{2} \xi^{1}=u^{2} / q^{2} \xi^{1} x^{2}-s u^{2} / q^{2} \xi^{3} x^{3}, \\
& x^{2} \xi^{3}=u / q \xi^{3} x^{2}, \quad x^{3} \xi^{2}=\left(u^{2} / q-1\right) \xi^{3} x^{2}+u \xi^{2} x^{3}, \\
& x^{1} \xi^{2}=\left(u^{2} / q-1\right) \xi^{1} x^{2}+q \xi^{2} x^{1}+s \xi^{3} x^{3} .
\end{aligned}
$$

The commutation relations $R_{\partial \xi}^{\Omega^{-1}}$ between the derivatives and the differentials :

$$
\begin{aligned}
& \partial_{1} \xi^{1}=q / u^{2} \xi^{1} \partial_{1}, \quad \partial_{3} \xi^{2} \quad=1 / u \xi^{2} \partial_{3}-s / q \xi^{3} \partial_{1}, \\
& \partial_{1} \xi^{3}=1 / u \xi^{3} \partial_{1}, \quad \partial_{2} \xi^{1} \quad=q^{2} / u^{2} \xi^{1} \partial_{2}, \\
& \partial_{2} \xi^{3}=q / u \xi^{3} \partial_{2}, \quad \quad \partial_{3} \xi^{1} \quad=q / u \xi^{1} \partial_{3}+s q / u^{2} \xi^{3} \partial_{2}, \\
& \partial_{1} \xi^{2}=1 / q \xi^{2} \partial_{1}, \quad \partial_{3} \xi^{3}=\left(q / u^{2}-1\right) \xi^{1} \partial_{1}+q / u^{2} \xi^{3} \partial_{3}, \\
& \partial_{2} \xi^{2}=\left(q / u^{2}-1\right) \xi^{1} \partial_{1}+\left(q / u^{2}-1\right) \quad \xi^{3} \partial_{3}+q / u^{2} \xi^{2} \partial_{2}
\end{aligned}
$$

The commutation relations $R_{x \partial}^{\Omega^{-1}}$ between the derivatives and the variables:

$$
\begin{aligned}
& \partial_{2} x^{2}=1+u^{2} / q x^{2} \partial_{2}, \quad \partial_{1} x^{3}=u / q x^{3} \partial_{1}, \\
& \partial_{3} x^{3}=1+u^{2} / q x^{3} \partial_{3}+\left(u^{2} / q-1\right) x^{2} \partial_{2}, \quad \partial_{2} x^{1} \quad=q x^{1} \partial_{2}, \\
& \partial_{1} x^{2}=u^{2} / q^{2} x^{2} \partial_{1}, \quad \partial_{3} x^{2} \quad=u / q x^{2} \partial_{3}-s u^{2} / q x^{3} \partial_{1}, \\
& \partial_{3} x^{1}=u x^{1} \partial_{3}+s x^{3} \partial_{2}, \quad \partial_{2} x^{3} \quad=u x^{3} \partial_{2}, \\
& \partial_{1} x^{1}=1+u^{2} / q x^{1} \partial_{1}+\left(u^{2} / q-1\right) x^{2} \partial_{2}+\left(u^{2} / q-1\right) x^{3} \partial_{3} .
\end{aligned}
$$

The two sets of relations $R_{x x}, R_{\xi \xi}, R_{\partial \partial}$ with $R^{\Omega}$ or $R^{\Omega^{-1}}$ define two quadratic algebras : $C<x, \xi, \partial>/ R \cup R^{\Omega}$ and $C<x, \xi, \partial>/ R \cup R^{\Omega^{-1}}$. In the following section, we investigate their invariance.

It is to be noted that all the construction of the differential calculus is performed without using the B-matrix associated with the variables [3] [ [4], and is the result solely of the relations $R_{x x}, R_{x \xi}$ and $R_{\partial \xi}$. Moreover, as a consequence of the construction, $B$ is found to be equal to $C$.

\section{Quantum Group and Invariance}

The quantum matrix $T$ with nine non commuting elements defines a homomorphism on $C<x, \xi, \partial>$ []]. The variables $x$ and the differentials $\xi$ are transformed by $T$ and the derivatives $\partial$ are transformed by $\left(T^{-1}\right)^{t}$. 
When the matrix $T$ satisfies

$$
R_{k l}^{j i} t_{m}^{k} t_{n}^{l}=t_{l}^{j} t_{k}^{i} R_{m n}^{l k}
$$

with $R=\Omega$ (resp. $R=\Omega^{-1}$ ), the relations $R \cup R^{\Omega}$ (resp. $R \cup R^{\Omega^{-1}}$ ) are invariant, and therefore this homomorphism maps $C<x, \xi, \partial>/ R \cup R^{\Omega}$ (resp. $C<x, \xi, \partial>/ R \cup R^{\Omega^{-1}}$ ) in itself. It is easy to see that $R=\Omega$ and $R=\Omega^{-1}$ define the same quantum matrix $T$, the elements of which satisfy the following commutation relations $R_{t t}$ :

$$
\begin{aligned}
& t_{2}^{1} t_{1}^{1}=q^{2} / u^{2} t_{1}^{1} t_{2}^{1}, \quad t_{2}^{2} t_{1}^{1}=t_{1}^{1} t_{2}^{2}-\left(u^{2}-q\right) / q^{2} t_{2}^{1} t_{1}^{2}-q s / u^{2} t_{1}^{3} t_{2}^{3}, \\
& t_{3}^{1} t_{2}^{1}=u / q t_{2}^{1} t_{3}^{1}, \quad t_{1}^{2} t_{1}^{1}=1 / q t_{1}^{1} t_{1}^{2}-s / q\left(t_{1}^{3}\right)^{2}, \\
& t_{3}^{1} t_{1}^{1}=q / u t_{1}^{1} t_{3}^{1}, \quad t_{3}^{2} t_{1}^{1}=u / q t_{1}^{1} t_{3}^{2}-\left(u^{2}-q\right) / q^{2} t_{3}^{1} t_{1}^{2}-s / q t_{3}^{3} t_{1}^{3}, \\
& t_{2}^{3} t_{2}^{2}=u t_{2}^{2} t_{2}^{3}, \quad t_{3}^{3} t_{1}^{1}=t_{1}^{1} t_{3}^{3}-\left(u^{2}-q\right) /(u q) t_{3}^{1} t_{1}^{3} \text {, } \\
& t_{1}^{3} t_{1}^{1}=1 / u t_{1}^{1} t_{1}^{3}, \quad t_{2}^{3} t_{1}^{1}=q / u t_{1}^{1} t_{2}^{3}-\left(u^{2}-q\right) / u t_{2}^{1} t_{1}^{3}, \\
& t_{3}^{3} t_{2}^{1}=1 / q t_{2}^{1} t_{3}^{3}, \quad t_{2}^{2} t_{2}^{1}=1 / q t_{2}^{1} t_{2}^{2}-s / q\left(t_{2}^{3}\right)^{2}, \\
& t_{3}^{2} t_{2}^{2}=u / q t_{2}^{2} t_{3}^{2}, \quad t_{3}^{2} t_{2}^{1}=u / q^{2} t_{2}^{1} t_{3}^{2}-s / q t_{3}^{3} t_{2}^{3}, \\
& t_{1}^{3} t_{2}^{1}=u / q^{2} t_{2}^{1} t_{1}^{3}, \quad t_{1}^{2} t_{2}^{1}=u^{2} / q^{3} t_{2}^{1} t_{1}^{2}-s / q t_{1}^{3} t_{2}^{3} \\
& t_{2}^{3} t_{1}^{2}=q^{2} / u t_{1}^{2} t_{2}^{3}, \quad t_{2}^{3} t_{3}^{1}=t_{3}^{1} t_{2}^{3}-\left(u^{2}-q\right) /(u q) t_{2}^{1} t_{3}^{3} \text {, } \\
& t_{2}^{3} t_{2}^{1}=1 / u t_{2}^{1} t_{2}^{3}, \quad t_{3}^{2} t_{3}^{1}=1 / q t_{3}^{1} t_{3}^{2}-s / q\left(t_{3}^{3}\right)^{2}+s / q t_{1}^{1} t_{2}^{2}-s u^{2} / q^{3} t_{2}^{1} t_{1}^{2}, \\
& t_{1}^{3} t_{3}^{1}=1 / q t_{3}^{1} t_{1}^{3}, \quad t_{2}^{2} t_{3}^{1}=u / q t_{3}^{1} t_{2}^{2}-\left(u^{2}-q\right) / q^{2} t_{2}^{1} t_{3}^{2}-s / u t_{3}^{3} t_{2}^{3}, \\
& t_{1}^{2} t_{2}^{2}=u^{2} / q^{2} t_{2}^{2} t_{1}^{2}, \quad t_{3}^{3} t_{2}^{2}=t_{2}^{2} t_{3}^{3}+\left(u^{2}-q\right) / u t_{3}^{2} t_{2}^{3} \\
& t_{3}^{2} t_{1}^{2}=q / u t_{1}^{2} t_{3}^{2}, \quad t_{3}^{3} t_{3}^{2}=u t_{3}^{2} t_{3}^{3}+s q / u t_{1}^{2} t_{2}^{3}-s u t_{2}^{2} t_{1}^{3} \text {, } \\
& t_{2}^{3} t_{1}^{3}=q^{2} / u^{2} t_{1}^{3} t_{2}^{3}, \quad t_{1}^{3} t_{2}^{2}=u / q t_{2}^{2} t_{1}^{3}+\left(u^{2}-q\right) / u t_{1}^{2} t_{2}^{3}, \\
& t_{2}^{3} t_{3}^{3}=q / u t_{3}^{3} t_{2}^{3}, \quad t_{1}^{3} t_{3}^{2}=t_{3}^{2} t_{1}^{3}+\left(u^{2}-q\right) / u t_{1}^{2} t_{3}^{3}, \\
& t_{1}^{3} t_{1}^{2}=u t_{1}^{2} t_{1}^{3}, \quad t_{1}^{2} t_{3}^{1}=u / q^{2} t_{3}^{1} t_{1}^{2}-s u / q^{2} t_{3}^{3} t_{1}^{3} \\
& t_{1}^{3} t_{3}^{3}=u / q t_{3}^{3} t_{1}^{3}, \quad t_{3}^{3} t_{3}^{1}=1 / u t_{3}^{1} t_{3}^{3}+s / u t_{1}^{1} t_{2}^{3}-s u / q^{2} t_{2}^{1} t_{1}^{3}, \\
& t_{3}^{3} t_{1}^{2}=q t_{1}^{2} t_{3}^{3}, \quad t_{2}^{3} t_{3}^{2}=q t_{3}^{2} t_{2}^{3}
\end{aligned}
$$

Any elements of $C\left\langle t>/ R_{t t}\right.$ can be written as a sum of ordered monomials $\left(t_{1}^{1}\right)^{k_{1}}\left(t_{2}^{1}\right)^{k_{2}}\left(t_{3}^{1}\right)^{k_{3}}\left(t_{2}^{2}\right)^{k_{4}}\left(t_{1}^{2}\right)^{k_{5}}\left(t_{3}^{2}\right)^{k_{6}}\left(t_{3}^{3}\right)^{k_{7}}\left(t_{1}^{3}\right)^{k_{8}}\left(t_{2}^{3}\right)^{k_{9}}$ by using the relations $R_{t t}$. The inverse $T^{-1}$, of $T$ is equal to :

$$
\left(\begin{array}{ccc}
t_{2}^{2} t_{3}^{3}-q / u t_{3}^{2} t_{2}^{3} & -q^{2} / u^{2} t_{2}^{1} t_{3}^{3}+q^{3} / u^{3} t_{3}^{1} t_{2}^{3} & t_{2}^{1} t_{3}^{2}-q / u t_{3}^{1} t_{2}^{2} \\
-u^{2} / q^{2} t_{1}^{2} t_{3}^{3}+u^{3} / q^{3} t_{3}^{2} t_{1}^{3} & t_{1}^{1} t_{3}^{3}-u / q t_{3}^{1} t_{1}^{3} & -u^{2} / q^{2} t_{1}^{1} t_{3}^{2}+u^{3} / q^{3} t_{3}^{1} t_{1}^{2} \\
t_{1}^{2} t_{2}^{3}-u^{2} / q^{2} t_{2}^{2} t_{1}^{3} & -q^{2} / u^{2} t_{1}^{1} t_{2}^{3}+t_{2}^{1} t_{1}^{3} & t_{1}^{1} t_{2}^{2}-u^{2} / q^{2} t_{2}^{1} t_{1}^{2}
\end{array}\right) D^{-1}
$$


with the determinant of $T$ equal to

$$
D=t_{1}^{1} t_{2}^{2} t_{3}^{3}+t_{3}^{1} t_{1}^{2} t_{2}^{3}+u^{3} / q^{3} t_{2}^{1} t_{3}^{2} t_{1}^{3}-q / u t_{1}^{1} t_{3}^{2} t_{2}^{3}-u^{2} / q^{2} t_{2}^{1} t_{1}^{2} t_{3}^{3}-u^{2} / q^{2} t_{3}^{1} t_{2}^{2} t_{1}^{3} .
$$

We can calculate and verify that $D$ is not a central element of $C<t>$ and therefore we have to add the generator $D^{-1}$ to this algebra. The commutation relations $R_{t D^{-1}}$ of $D^{-1}$ with all the $t_{j}^{i}$ are deduced from those of $D$ :

$$
\begin{aligned}
t_{1}^{1} D^{-1} & =D^{-1} t_{1}^{1}, & t_{2}^{1} D^{-1} & =u^{2} / q^{4} D^{-1} t_{2}^{1}, & t_{3}^{1} D^{-1} & =u / q^{2} D^{-1} t_{3}^{1}, \\
t_{2}^{2} D^{-1} & =D^{-1} t_{2}^{2}, & t_{1}^{2} D^{-1} & =q^{2} D^{-1} t_{1}^{2}, & t_{3}^{2} D^{-1} & =u / q^{2} D^{-1} t_{3}^{2}, \\
t_{1}^{3} D^{-1} & =q^{2} / u D^{-1} t_{1}^{3}, & t_{2}^{3} D^{-1} & =u / q^{2} D^{-1} t_{2}^{3}, & t_{3}^{3} D^{-1} & =D^{-1} t_{3}^{3} .
\end{aligned}
$$

The quotient algebra $C<t, D^{-1}>/ R_{t t} \cup R_{t D^{-1}}$ is a Hopf algebra with the co-product $\Delta$, the co-unit $\epsilon$ and antipode $S$ defined by :

$$
\begin{array}{r}
\Delta(T) \equiv T \otimes T, \quad \Delta\left(D^{-1}\right) \equiv D^{-1} \otimes D^{-1} \\
\epsilon\left(T, D^{-1}\right) \equiv(I, 1), \quad S(T) \equiv T^{-1}, \quad S(D) \equiv D^{-1}
\end{array}
$$

When $x^{1}$ and $x^{2}$ are mutually adjoint and when $x^{3}$ is self-adjoint (for instance, in the case of the q-oscillator algebra), the relations $R_{x x}$ are unchanged if the parameters are real. The action of $T$ respects this property if the quantum group is equipped with a star-operation that is an antihomomorphism such as

$$
\begin{array}{rrrr}
\left(\left(t_{j}^{i}\right)^{*}\right)^{*} & =t_{j}^{i}, & \forall i, j \\
\left(t_{2}^{2}\right)^{*}=t_{1}^{1}, & \left(t_{1}^{2}\right)^{*} & =t_{2}^{1}, & \left(t_{3}^{2}\right)^{*}=t_{3}^{1}, \\
\left(t_{1}^{3}\right)^{*} & =t_{2}^{3}, & \left(t_{3}^{3}\right)^{*}=t_{3}^{3}
\end{array}
$$

These relations are consistent with (22) and (24) and the quantum group $C<t, D^{-1}>/ R_{t t} \cup R_{t D^{-1}}$ acquires the structure of a Hopf-star-algebra.

Let us stress some properties of $C<t, D^{-1}>/ R_{t t} \cup R_{t D^{-1}}$ :

- When $s=0$, the variable quantum space is the three-dimensional quantum plane, the resulting quantum group corresponds to an original deformation of $G L(3)$ [7] , [6].

- When $t_{1}^{3}$ and $t_{2}^{3}$ vanish, we obtain a deformation $G_{q s}$ of the subgroup $G$ of $G L(3)$. Two of the relations $R_{t t}$ give :

$$
\left(u^{2}-q\right) t_{2}^{1} t_{3}^{3}=0
$$


and

$$
\left(u^{2}-q\right) t_{1}^{2} t_{3}^{3}=0 \text {. }
$$

implying that $q$ is equal to $u^{2}$ if the algebra has no zero divisors. When $q=u^{2}$, the matrix $\Omega$ being equal to its inverse, the two differential calculi on $C<$ $x>/ R_{x x}$ reduce to one. This differential calculus was previously obtained by a completely different method, implying the uniqueness of the result [11]. All the commutation relations are invariant under the ten-generator quantum group $C<t, D^{-1}>/ R_{t t} \cup R_{t D^{-1}}$ and under a quantum group $G_{q s}$ deduced from the previous one by putting $t_{1}^{3}=t_{2}^{3}=0$.

- The relations (27) are consistent with the condition $t_{1}^{3}=t_{2}^{3}=0$, and $G_{q s}$ is a Hopf-star-subalgebra of $C<t, D^{-1}>/ R_{t t} \cup R_{t D^{-1}}$.

- In the case where $q=u^{2}$, we would point out that, if we add the generator $\left(t_{3}^{3}\right)^{-1}$ to the quantum group $G_{q s}$, the $T$-matrix can be written on the form $T^{\prime} \times t_{3}^{3}$ with $t_{j}^{i}=t_{j}^{i}\left(t_{3}^{3}\right)^{-1}$. All the elements $t_{j}^{i}$ commute two by two. Nevertheless, they cannot be identified to C-numbers (and then $T^{\prime}$ to a matrix belonging to the initial subgroup $G$ ), because if they were C-numbers all the elements $t_{j}^{i}$ would be proportional to $t_{3}^{3}$ and this is impossible due to their commutation relations resulting from (22).

\section{Conclusion}

Two differential calculi can be associated with the three-parameter oscillator algebra $C<x>/ R_{x x}$ and in particular with the q-oscillator algebra. They are invariant under the same quantum group $C<t, D^{-1}>/ R_{t t} \cup R_{t D^{-1}}$ that is an original three-parameter deformation of $G L(3)$. When we assume that the variables $x^{1}$ and $x^{2}$ are mutually adjoint and that $x^{3}$ is self adjoint, a star-operation is defined on the invariance quantum group that then becomes a Hopf-star-algebra. Finally we consider the case where two generators, $t_{1}^{3}$ and $t_{2}^{3}$, are removed from the algebra $C<t, D^{-1}>/ R_{t t} \cup R_{t D^{-1}}$ and we prove that the resulting differential calculus and quantum group of invariance do not exist for arbitrary values of the parameters, this is in agreement with a previous work [10] 11].

We would like to thank J.Bertrand for the many interesting discussions we have had. 


\section{References}

[1] Woronowicz, S.L.(1989) Differential Calculus on Compact Matrix Pseudogroups (Quantum Groups) Commun. Math. Phys, 122, pp. 125-170.

[2] Bernard, D. (1990) Quantum Lie Algebras and Differential Calculus on Quantum Groups Progress of Theoretical Physics Supplement 102 pp. 49-66.

[3] Wess, J. and Zumino, B. (1990) Covariant Differential Calculus on the Quantum Hyperplane Nucl. Phys. (Proc. Suppl.), B 18, pp. 301-313.

[4] Zumino, G. (1992) Differential calculus on quantum spaces and quantum groups, in Group Theoretical Methods in Physics, M.A. del Olmo, M.Santander and J.Mateos Guilarte (Eds.), Anales de Física Monografias, CIEMAT (Spain), pp. 41-59.

[5] Manin, Yu. I. (1988) Quantum groups and non-commutative geometry, (Publications du CRM, Université de Montréal, Montréal).

[6] Schirrmacher, A. (1991) The Multiparametric Deformation of GL(n) and the Covariant Differential calculus on the quantum vector space, Z. Phys. C, 50, pp 321-327.

[7] Reshetikhin, N.Yu., Takhtadzhyan, L.A. and Faddeev, L.D. (1990) Quantization of Lie groups and Lie algebras, Leningrad Math. J., 1, pp. 193-225.

[8] Mac Farlane, A.J. (1989) On q-analogues of the quantum harmonic oscillator and the quantum group $S U(2)_{q}$, J.Phys. A, 22, p. L 4581.

[9] Biedenharn, L.C. (1989) The quantum group $S U_{q}(2)$ and a $q$-analogue of the boson operators J.Phys. A, 22, p. L 873.

[10] Bertrand, J. and Irac-Astaud, M. (1995) Invariant differential calculus on a deformation of the Weyl-Heisenberg algebra, Modern Group Theoretical Methods in Physics,p. 37 Kluwer Academic Publishers.

[11] Bertrand, J. and Irac-Astaud, M.(1996) Invariance quantum groups of a deformed oscillator algebra, Preprint LPTM 